\title{
Double-Pionic Fusion of Nuclear Systems and the "ABC" Effect: Approaching a Puzzle by Exclusive and Kinematically Complete Measurements
}

\author{
M. Bashkanov, ${ }^{1}$ C. Bargholtz, ${ }^{11}$ M. Berłowski, ${ }^{6}$ D. Bogoslawsky, ${ }^{2}$ H. Calén, ${ }^{3}$ H. Clement, ${ }^{1}$ L. Demiroers,${ }^{5}$
}

E. Doroshkevich, ${ }^{1}$ D. Duniec, ${ }^{4}$ C. Ekström, ${ }^{3}$ K. Fransson, ${ }^{3}$ L. Geren, ${ }^{11}$ L. Gustafsson, ${ }^{4}$ B. Höistad, ${ }^{4}$ G. Ivanov, ${ }^{2}$ M. Jacewicz, ${ }^{4}$ E. Jiganov, ${ }^{2}$ T. Johansson, ${ }^{4}$ O. Khakimova, ${ }^{1}$ S. Keleta ${ }^{4}$ I. Koch,${ }^{4}$ F. Kren, ${ }^{1}$ S. Kullander, ${ }^{4}$ A. Kupść, ${ }^{3}$ K. Lindberg, ${ }^{11}$ P. Marciniewski, ${ }^{3}$ R. Meier, ${ }^{1}$ B. Morosov, ${ }^{2}$ C. Pauly, ${ }^{7}$ H. Pettersson, ${ }^{4}$ Y. Petukhov, ${ }^{2}$ A. Povtorejko, ${ }^{2}$ A. Pricking, ${ }_{1}^{1}$ R. J. M. Y. Ruber, ${ }^{3}$ K. Schönning, ${ }^{4}$ W. Scobel,${ }^{5}$ B. Shwartz, ${ }^{8}$ T. Skorodko, ${ }^{1}$ V. Sopov, ${ }^{10}$ J. Stepaniak, ${ }^{6}$ P.-E. Tegner, ${ }^{11}$ P. Thörngren-Engblom, ${ }^{4}$ V. Tikhomirov, ${ }^{2}$ A. Turowiecki, ${ }^{9}$ G. J. Wagner, ${ }^{1}$ M. Wolke, ${ }^{7}$ J. Zabierowski, ${ }^{7}$ I. Zartova, ${ }^{11}$ and J. Złomanczuk ${ }^{4}$

\section{(CELSIUS/WASA Collaboration)}

\author{
${ }^{1}$ Physikalisches Institut der Universität Tübingen, D-72076 Tübingen, Germany \\ ${ }^{2}$ Joint Institute for Nuclear Research, Dubna, Russia \\ ${ }^{3}$ The Svedberg Laboratory, Uppsala, Sweden \\ ${ }^{4}$ Uppsala University, Uppsala, Sweden \\ ${ }^{5}$ Hamburg University, Hamburg, Germany \\ ${ }^{6}$ Soltan Institute of Nuclear Studies, Warsaw and Lodz, Poland \\ ${ }^{7}$ Forschungszentrum Jülich, Germany \\ ${ }^{8}$ Budker Institute of Nuclear Physics, Novosibirsk, Russia \\ ${ }^{9}$ Institute of Experimental Physics, Warsaw, Poland \\ ${ }^{10}$ Institute of Theoretical and Experimental Physics, Moscow, Russia \\ ${ }^{11}$ Department of Physics, Stockholm University, Stockholm, Sweden
}

(Received 1 July 2008; revised manuscript received 10 October 2008; published 2 February 2009)

\begin{abstract}
The ABC effect-a puzzling low-mass enhancement in the $\pi \pi$ invariant mass spectrum, first observed by Abashian, Booth, and Crowe-is well known from inclusive measurements of two-pion production in nuclear fusion reactions. Here we report on the first exclusive and kinematically complete measurements of the most basic double-pionic fusion reaction $p n \rightarrow d \pi^{0} \pi^{0}$ at beam energies of 1.03 and $1.35 \mathrm{GeV}$. The measurements, which have been carried out at CELSIUS-WASA, reveal the ABC effect to be a $(\pi \pi)_{I=L=0}$ channel phenomenon associated with both a resonancelike energy dependence in the integral cross section and the formation of a $\Delta \Delta$ system in the intermediate state. A corresponding simple $s$-channel resonance ansatz provides a surprisingly good description of the data.
\end{abstract}

The ABC effect-first observed by Abashian, Booth, and Crowe [1] in the double-pionic fusion of deuterons and protons to ${ }^{3} \mathrm{He}$ - stands for an unexpected enhancement at low masses in the invariant $\pi \pi$ mass spectrum $M_{\pi \pi}$. Follow-up experiments [2-11] revealed this effect to be of an isoscalar nature and to show up in cases when the two-pion production process leads to a bound nuclear system. With the exception of low-statistics bubblechamber measurements [4,8], all experiments conducted on this issue have been inclusive measurements carried out preferentially with single-arm magnetic spectrographs for the detection of the fused nuclei.

Initially, the low-mass enhancement had been interpreted as an unusually large $\pi \pi$ scattering length and evidence for the $\sigma$ meson, respectively [1]. Since the effect showed up particularly clear at beam energies corresponding to the excitation of two $\Delta s$ in the nuclear system, the $\mathrm{ABC}$ effect was interpreted later on by a $t$-channel $\Delta \Delta$ excitation in the course of the reaction process leading to both a low-mass and a high-mass enhancement in isoscalar $M_{\pi \pi}$ spectra [12-17]. In fact, the missing momentum spectra from inclusive measurements have been in support of such predictions.

In order to shed more light on this issue and complementing our previous result on the double-pionic fusion to ${ }^{3} \mathrm{He}[18,19]$, exclusive and kinematically overconstrained measurements have been carried out on the most basic system for double-pionic fusion, the $p n \rightarrow d \pi^{0} \pi^{0}$ reaction. To this end, we have measured this reaction in the quasifree mode $p d \rightarrow p_{\text {spectator }} d \pi^{0} \pi^{0}$ at beam energies $T_{p}=1.03$ and $1.35 \mathrm{GeV}$ at CELSIUS using the $4 \pi$ WASA detector setup including the pellet target system [20]. The latter provides frozen deuterium pellets of size $\approx 20 \mu \mathrm{m}$, which cross the beam perpendicularly with a frequency of $\approx 7 \mathrm{kHz}$. The beam energies have been chosen to be in the region of the $\mathrm{ABC}$ effect as known 
from inclusive measurements. The experimental results on the $p d \rightarrow{ }^{3} \mathrm{He} \pi^{0} \pi^{0}$ and $p d \rightarrow{ }^{3} \mathrm{He} \pi^{+} \pi^{-}$reactions and first results from the measurements of the $d d \rightarrow{ }^{4} \mathrm{He} \pi \pi$ reaction are given in Refs. [18,19,21].

The deuterons emerging from the reaction of interest here have been detected in the forward detector and identified by the $\Delta E-E$ technique using corresponding information from a quirl and range hodoscope, respectively (see, e.g., Fig. 1 of Ref. [18]). $\gamma$ rays from the $\pi^{0}$ decay have been detected in the central detector.

This way, the full four-momenta have been measured for all particles of an event-except for the very low-energetic spectator proton, which did not reach any active detector element. Thus kinematic fits with 3 overconstraints could be performed for each event.

Because of Fermi motion of the nucleons in the target deuteron, the quasifree reaction proceeds via a range of effective collision energies. Based on the measured energies in the exit channel and the thus event-by-event reconstructed total energies in the $p n$ system, the data have been binned into small ranges of effective beam energy, in order to reduce the kinematical smearing and also to allow the extraction of the energy dependence in the total cross section.

The absolute normalization of these data has been obtained by normalization relative to the quasifree singlepion production $p n \rightarrow d \pi^{0}$ [22] measured simultaneously with the same particle trigger. For the higher beam energy, we used in addition the $p n \rightarrow d \eta$ production process for an alternative calibration. Both calibration methods agree within $10 \%$. However, since at the higher energy the phase-space coverage is less complete than at the lower energy, the determination of the absolute cross section depends somewhat on the model used in the MC simulation for acceptance and efficiency correction. Hence the cross sections derived for the higher beam energy are plotted in Fig. 4 with an increased uncertainty. Also, in order to avoid contaminations due to the increased $\pi \pi \pi$ production and other background, we introduced in the analysis of the high energy data kinematic constraints on the spectator proton before kinematic fits.
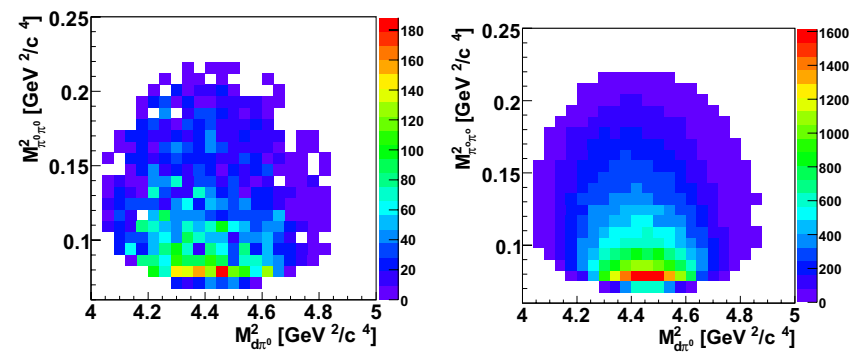

Results of our measurements are shown in Figs. 1-4. Since the two $\pi^{0}$ particles emerging from the reaction of interest are identical particles, observables depending only on a single $\pi^{0}$ like $M_{d \pi^{0}}$ are calculated by averaging over both possible combinations. Figure 1 displays the Dalitz plots of the invariant mass squares $M_{d \pi^{0}}^{2}$ versus $M_{\pi^{0} \pi^{0}}^{2}$ and of the invariant mass square $M_{d \pi^{0}}^{2}$ of the deuteron with one of the pions versus the same quantity with the other pionfor both data and Monte Carlo (MC) simulations of a model ansatz discussed below. The Dalitz plots are far from being flat, i.e., far from being phase-space-like. They rather exhibit very pronounced enhancements in the region of the $\Delta$ resonance and at the low-mass kinematic limit of $M_{\pi^{0} \pi^{0}}^{2}$. This unusual phenomenon is known as the $\mathrm{ABC}$ effect. In this reaction the $\pi^{0} \pi^{0}$ channel is free of both isovector $(I=1)$ and isotensor $(I=2)$ contributions due to vanishing isospin coupling coefficients. Hence the observation of the $\mathrm{ABC}$ effect here means that it must be of isoscalar $(I=0)$ character. Figure 2 depicts the Dalitz-plot projections, the spectra of invariant mass squares $M_{\pi^{0} \pi^{0}}^{2}$ and $M_{d \pi^{0}}^{2}$ for the two bins of effective collision energies $T_{p}=1.00-1.03 \mathrm{GeV}$ and $T_{p}=1.34-1.37 \mathrm{GeV}$, respectively. At both energies, low-mass $M_{\pi^{0} \pi^{0}}$ enhancement and $\Delta$ excitation, respectively, are the predominating structures.

Angular distributions are shown in Fig. 3. The distribution of the opening angle $\delta_{\pi^{0} \pi^{0}}^{d \pi^{0}}$ between the two pions in the $d \pi^{0} \pi^{0}$ (or, equivalently, pn) center-of-mass (c.m.) system peaks at small angles, in particular, if we select events with $M_{\pi^{0} \pi^{0}} \leq 0.32 \mathrm{GeV} / c^{2}$. This means that the low-mass enhancement is associated with pions leaving the interaction vertex in parallel. The distributions of the deuteron polar angles $\Theta_{d}^{d \pi^{0} \pi^{0}}$ in the $d \pi^{0} \pi^{0}$ c.m. system and of the pion polar angles $\Theta_{\pi^{0}}^{\pi^{0} \pi^{0}}$ and $\Theta_{\pi^{0}}^{d \pi^{0}}$ in the $\pi^{0} \pi^{0}$ and $d \pi^{0}$ subsystems, respectively, are anisotropic and essentially symmetric about $90^{\circ}$. The anisotropy observed for the latter corresponds just to the one expected from $\Delta$ decay. The anisotropy in the $\pi^{0}$ angular distribution in the $\pi \pi$ subsystem signals some $d$-wave admixture. It vanishes, if we consider only data with $M_{\pi^{0} \pi^{0}} \leq 0.32 \mathrm{GeV} / c^{2}$, i.e., in
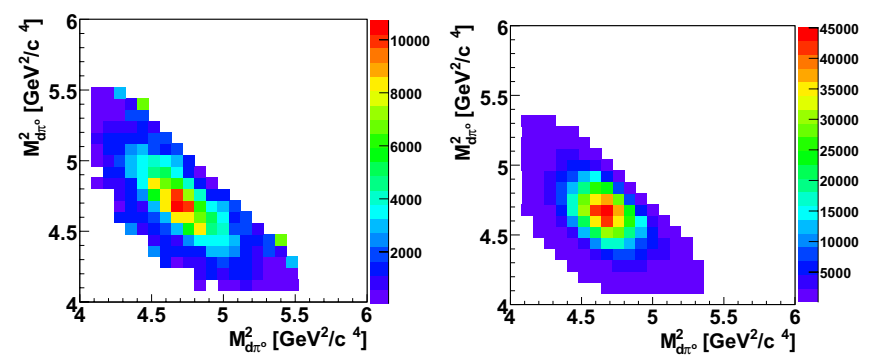

FIG. 1 (color online). Dalitz plot of the invariant mass distributions for $M_{d \pi^{0}}^{2}$ versus $M_{\pi^{0} \pi^{0}}^{2}$ (first and second on the left: data and MC simulation, respectively, for collision energy $1.00-1.03 \mathrm{GeV}$ ) and $M_{d \pi^{0}}^{2}$ versus $M_{d \pi^{0}}^{2}$ (first and second on the right: data and MC simulation, respectively, for collision energy $1.27-1.37 \mathrm{GeV})$ for the quasifree reaction process $p n \rightarrow d \pi^{0} \pi^{0}$. The color-coded scale $(z$ axis) is in arbitrary units. 

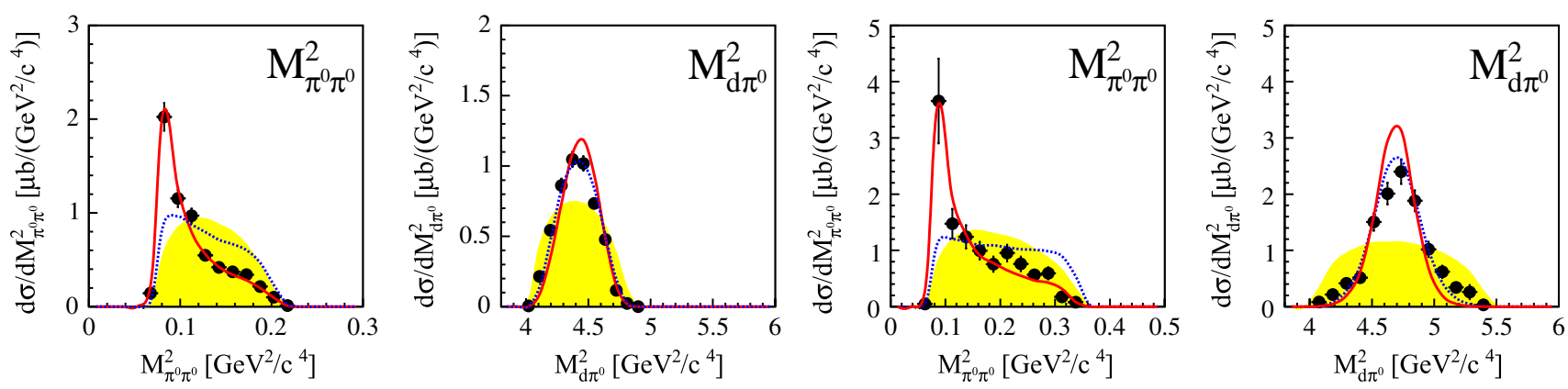

FIG. 2 (color online). Distributions of the invariant masses $M_{\pi^{0} \pi^{0}}$ and $M_{d \pi^{0}}$ from the exclusive measurements (full dots) of the $p n \rightarrow d \pi^{0} \pi^{0}$ reaction at effective collision energies of 1.00-1.03 (left) and 1.34-1.37 GeV (right). The shaded areas show the pure phase-space distributions. Solid and dotted curves give $\Delta \Delta$ calculations with and without the assumption of an $s$-channel resonance, respectively. All curves are normalized to the experimental integral cross section.

the region of the low-mass enhancement. From this we deduce that the enhancement is of a scalar nature, i.e., in total of a scalar-isoscalar nature.

The observed low-mass enhancement is consistent with the findings in previous inclusive single-arm measurements, where only the momentum of outgoing fused nuclei was measured. Note that the momentum of the fused ejectile is directly related to the invariant $\pi \pi$ mass in the restricted kinematical range of the measured scattering angle (see Figs. 3-5 in Ref. [18] and Refs. [1-7]).

The momentum spectra of inclusive single-arm measurements [7,9] exhibit also a strong central maximum (corresponding to large $M_{\pi \pi}$ values), if the deuteron scattering angle $\Theta_{d}^{\text {lab }}$ is close to $0^{\circ}$. A possible albeit controversial explanation has been given in Ref. [9] by associating this central maximum with $\eta$ and $\pi \pi \pi$ production. In our $M_{\pi \pi}$ data, which cover all angles $\Theta_{d}^{\text {lab }} \geq$ $3^{\circ}$, we observe no apparent high-mass enhancement.

The $\pi \pi$ low-mass enhancements observed in the exclusive data for the $\pi^{0} \pi^{0}$ channels are much larger than predicted in previous $\Delta \Delta$ calculations [12-16]. As an example, we show in Figs. 2-4 (dashed lines) calculations in the model ansatz of Ref. [12], where we additionally included the pion angular distribution in $\Delta$ decay and the
Fermi smearing of the nucleons bound in the final nucleus. We have chosen this model ansatz, since in addition to its simplicity it gives the smallest high-mass enhancement, i.e., is closest to our observation in this respect.

Since, on the one hand, the available $\Delta \Delta$ calculations obviously fail, but, on the other hand, the data clearly show the $\Delta \Delta$ excitation in their $M_{N \pi}$ spectra, a profound physics piece appears to be missing in the interpretation. As we demonstrate in the following, such a missing piece is found by inspection of the energy dependence of the total cross section displayed in Fig. 4. Shown are the results of this work (solid circles) for $p n \rightarrow d \pi^{0} \pi^{0}$-multiplied by the isospin factor of 2-in comparison with those of Refs. [4,8] for the $p n \rightarrow d \pi^{+} \pi^{-}$reaction (open symbols). The latter reaction is composed of $I=0$ and 1 contributions. The isovector part can be directly derived from the known $p p \rightarrow d \pi^{+} \pi^{0}$ cross sections by use of isospin relations [22]. Since the $p p \rightarrow d \pi^{+} \pi^{0}$ reaction is very well described [23] by conventional $t$-channel calculations for the formation of a $\Delta \Delta$ system in the intermediate state, also the isoscalar part of this conventional process can be derived by applying isospin relations to the intermediate $\Delta \Delta$ system. The thus obtained $I=0$ part is shown in Fig. 4 by the dotted line, whereas the noninterfering sum of
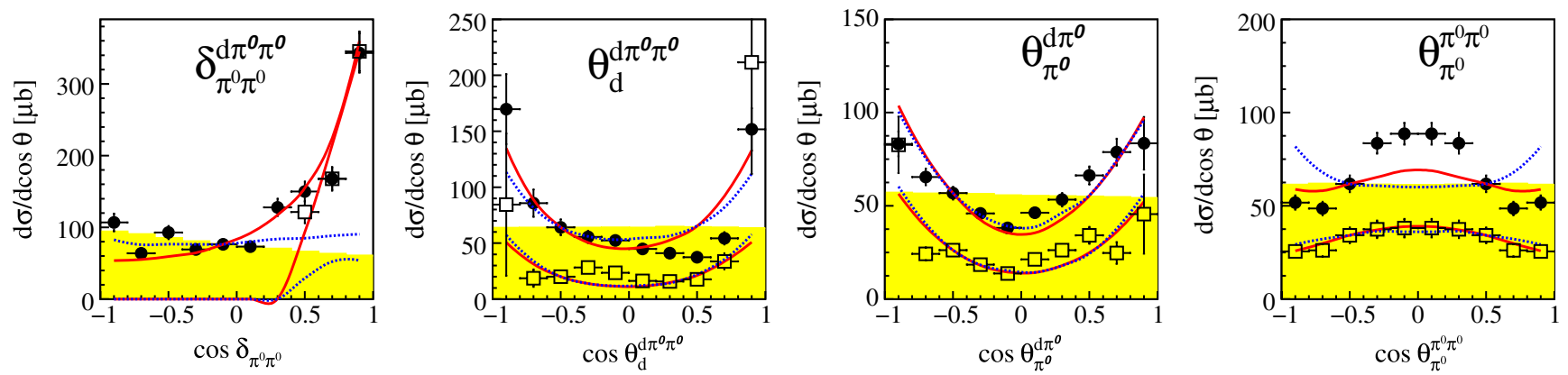

FIG. 3 (color online). Angular distributions in the reaction $p n \rightarrow d \pi^{0} \pi^{0}$ at $T_{p}=1.03 \mathrm{GeV}$ for the opening angle $\delta_{\pi^{0}}^{d \pi^{0} \pi 0} \pi^{0}$ between the two pions, the angle of the deuteron $\Theta_{d}^{d \pi^{0} \pi 0}$ —all in the $d \pi^{0} \pi^{0}$ c.m. system — as well as the pion angles $\Theta_{\pi^{0}}^{d \pi^{0}}$ and $\Theta_{\pi}^{\pi \pi \pi^{0}}$ in the $d \pi^{0}$ and $\pi \pi$ subsystems (Jackson frame), respectively. The meaning of symbols and curves is as in Fig. 2. In addition, data (open squares) and calculations are plotted also with the constraint $M_{\pi \pi}<0.32 \mathrm{GeV} / c^{2}$. 


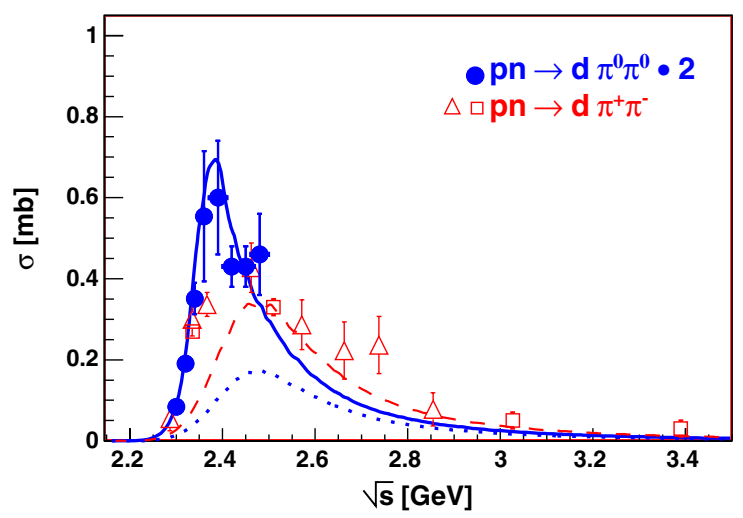

FIG. 4 (color online). Energy dependence of the total cross section for the $p n \rightarrow d \pi^{+} \pi^{-}$reaction from threshold $(\sqrt{s}=$ $2.15 \mathrm{GeV}$ ) up to $\sqrt{s}=3.5 \mathrm{GeV}$. Experimental data are from Refs. [8] (open squares) and [4] (open triangles). The results of this work for the $\pi^{0} \pi^{0}$ channel-scaled by the isospin factor of 2 - are given by the full circles. Dashed and dotted lines represent the cross sections for $\pi^{+} \pi^{-}$and $\pi^{0} \pi^{0}$ channels, respectively, as expected from the isovector $\pi^{+} \pi^{0}$ data by isospin relations (see text). The solid curve includes an $s$-channel resonance in the $\Delta \Delta$ system adjusted to describe the ABC effect in the $\pi^{0} \pi^{0}$ channel.

isoscalar and isovector parts is given by the dashed line. Indeed, the $\pi^{+} \pi^{-}$data are in reasonable agreement with this sum for $\sqrt{s} \geq 2.5 \mathrm{GeV}$. At lower energies, however, the measured values are much larger than expected from the conventional $\Delta \Delta$ process.

The so far unexplained structure observed for $\sqrt{s}<$ $2.5 \mathrm{GeV}$ in our data is much larger and narrower than expected from the conventional $\Delta \Delta$ excitation by $t$-channel meson exchange $[12,13,16]$. The cross section maximum is shifted substantially below the nominal $\Delta \Delta$ threshold being, however, still $200 \mathrm{MeV}$ above the $d \pi \pi$ threshold.

Since isospin is very unlikely to be broken on such a large scale, a mechanism different from the $t$-channel $\Delta \Delta$ excitation must be the reason for this resonancelike structure in the isoscalar sector. These considerations led us to the concept of an $s$-channel resonance, which couples to isoscalar $p n$ and $\Delta \Delta$ configurations. We note that such a kind of dibaryon resonance has been predicted by a number of theoretical investigations [24-29].

For the description of the observables by such a resonance, we use the following Breit-Wigner ansatz for the resonance amplitude:

$$
A_{R} \sim F\left(q_{\Delta \Delta}\right) D_{R} D_{\Delta_{1}} D_{\Delta_{2}},
$$

with the mass and width of the $s$-channel resonance being $m_{R} \approx 2.36 \mathrm{GeV} / c^{2}$ and $\Gamma_{R} \approx 80 \mathrm{MeV}$, respectively, and where $D_{R}$ and $D_{\Delta}$ stand for $s$-channel resonance and $\Delta$ propagators, respectively. The form factor $F\left(q_{\Delta \Delta}\right)$ of the $\Delta \Delta$ vertex, which is chosen to be of monopole type, depends on the relative momentum $q_{\Delta \Delta}$ between the two
$\Delta s$. Since $q_{\Delta \Delta}=q_{\pi \pi}$, when neglecting the Fermi motion of the nucleons, this form factor is reflected directly in the $M_{\pi \pi}$ spectra and causes there the ABC effect by suppression of the high-mass region. Fitting the cutoff parameter $\Lambda_{\Delta \Delta}$ of this monopole form factor to the data in the $M_{\pi \pi}$ spectra results in $\Lambda_{\Delta \Delta} \approx 0.2 \mathrm{GeV} / c$, which corresponds to the size of a deuteronlike object.

While other theoretical explanations cannot be ruled out at this time, with such a simple ansatz we obtain a surprisingly good description of the data (MC Dalitz plot in Fig. 1 and solid lines in Figs. 2-4) both in their energy dependence and in their differential behavior. In previous attempts $[18,30]$ of data interpretations, we had to impose a strong attraction or even a bound state condition between the two $\Delta$ 's, in order to obtain a satisfactory description of data on the $A B C$ effect. Having learned now from the behavior of the total cross section, that possibly an $s$-channel resonance could be the cause, these impositions would find a natural explanation.

We finally note that, after the move of the WASA detector to COSY, new data have been taken there very recently on this issue with 2 orders of magnitude higher statistics. It is expected that these will be able to finally solve the long-standing $\mathrm{ABC}$ puzzle.

We acknowledge valuable discussions with L. AlvarezRuso, V. Anisovich, D. Bugg, L. Dakhno, C. Hanhart, M. Kaskulov, V. Kukulin, E. Oset, I. Strakovsky, F. Wang, W. Weise, and C. Wilkin on this issue. This work has been supported by BMBF (No. 06TU201 and No. 06TU261), COSY-FFE, DFG (European Graduiertenkolleg 683), and the Swedish Research Council.

[1] N. E. Booth, A. Abashian, and K. M. Crowe, Phys. Rev. Lett. 7, 35 (1961); 5, 258 (1960); Phys. Rev. 132, 2296 (1963).

[2] R. J. Homer et al., Phys. Lett. 9, 72 (1964).

[3] J. H. Hall et al., Nucl. Phys. B12, 573 (1969).

[4] I. Bar-Nir et al., Nucl. Phys. B54, 17 (1973).

[5] J. Banaigs et al., Nucl. Phys. B67, 1 (1973).

[6] J. Banaigs et al., Nucl. Phys. B105, 52 (1976).

[7] F. Plouin et al., Nucl. Phys. A302, 413 (1978).

[8] A. Abdivaliev et al., Sov. J. Nucl. Phys. 29, 796 (1979); Nucl. Phys. B168, 385 (1980).

[9] F. Plouin, P. Fleury, and C. Wilkin, Phys. Rev. Lett. 65, 690 (1990).

[10] R. Wurzinger et al., Phys. Lett. B 445, 423 (1999).

[11] For a review, see A. Codino and F. Plouin, Report No. LNS/Ph/94-06.

[12] T. Risser and M. D. Shuster, Phys. Lett. 43B, 68 (1973).

[13] I. Bar-Nir, T. Risser, and M. D. Shuster, Nucl. Phys. B87, 109 (1975).

[14] J. C. Anjos, D. Levy, and A. Santoro, Nucl. Phys. B67, 37 (1973).

[15] See, e.g., A. Gardestig, G. Fäldt, and C. Wilkin, Phys. Rev. C 59, 2608 (1999); Phys. Lett. B 421, 41 (1998). 
[16] C. A. Mosbacher and F. Osterfeld, nucl-th/9903064.

[17] L. Alvarez-Ruso, Phys. Lett. B 452, 207 (1999); Ph.D. thesis, Universitat Valencia, 1999.

[18] M. Bashkanov et al., Phys. Lett. B 637, 223 (2006).

[19] M. Bashkanov, Ph.D. thesis, Universität Tübingen, 2006.

[20] Chr. Bargholtz et al., Nucl. Instrum. Methods Phys. Res., Sect. A 594, 339 (2008).

[21] S. Keleta, Ph.D. thesis, Uppsala Universitet, 2008.

[22] J. Bystricky et al., J. Phys. (Paris) 48, 1901 (1987).

[23] F. Kren et al., in Proceedings of the 10th International Workshop on Meson Production, Properties and Interaction (MESON08) (to be published).
[24] J. Ping et al., Phys. Rev. C 65, 044003 (2002), and references therein; arXiv:0806.0458.

[25] T. Barnes et al., Phys. Rev. C 48, 539 (1993).

[26] T. Kamae and T. Fujita, Phys. Rev. Lett. 38, 471 (1977).

[27] M. Oka and K. Yazaki, Prog. Theor. Phys. 66, 572 (1981).

[28] V. I. Kukulin et al., Nucl. Phys. A689, 327 (2001).

[29] R. D. Mota et al., Phys. Rev. C 65, 034006 (2002).

[30] M. Bashkanov et al., in Proceedings of the 11th International Conference on Meson-Nucleon Physics and the Structure of the Nucleon (MENU 2007), eConf C070910, 129 (2007). 